\title{
Partial Unfolding of Tubulin Heterodimers Induced by Two-Photon Excitation of Bound Meso-
}

tetrakis (Sulfonatophenyl) Porphyrin

Brady McMicken, ${ }^{\mathrm{a}, \mathrm{b}}$ Robert J. Thomas, ${ }^{\mathrm{b}}$ Lorenzo Brancaleon* ${ }^{\mathrm{a}}$

${ }^{a}$ The University of Texas at San Antonio, Department of Physics and Astronomy, One UTSA Circle, San Antonio, Texas, 78249, United States

${ }^{b}$ 711th Human Performance Wing, Human Effectiveness Directorate, Bioeffects Division, Optical Radiation Bioeffects Branch, JBSA Fort Sam Houston, Texas 78234, United States

\section{Supplemental Information.}

The figures below are analogous, respectively, to Figure 4A, Figure 4B and Figure 5.

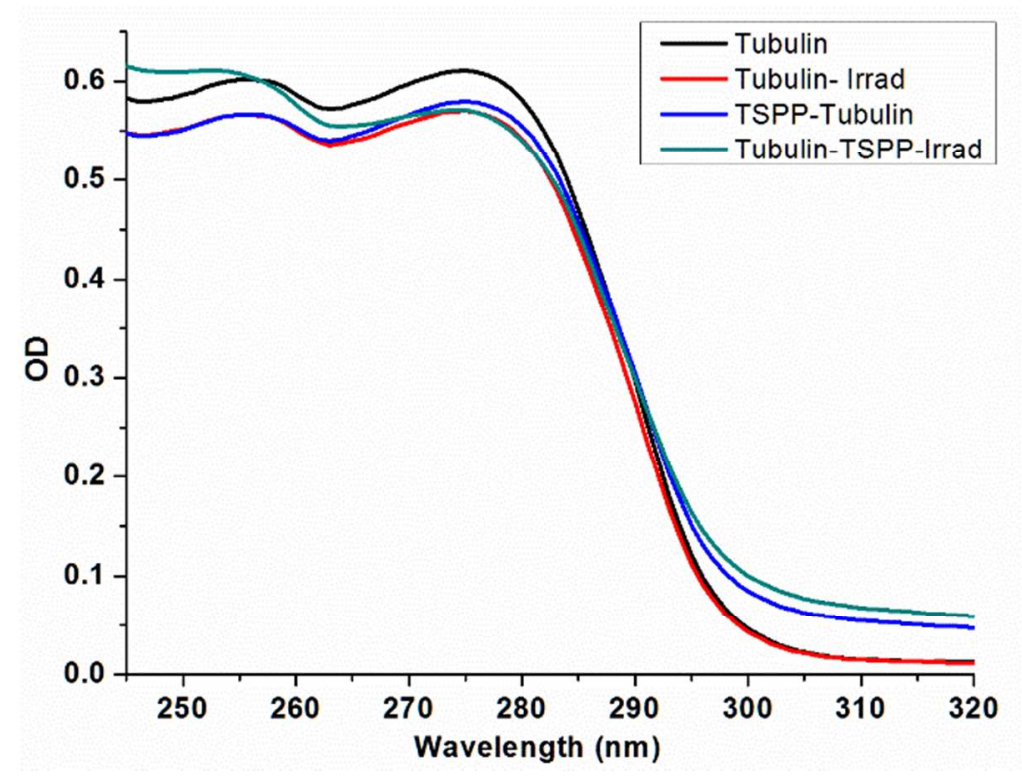

Figure S1. The effects of irradiation with the 80-MHz source on tubulin/TSPP complex. Absorption spectra of tubulin with tubulin alone (black), tubulin irradiated (red), TSPP/tubulin non-irradiated (blue), TSPP/tubulin complex irradiated (teal). (B) Fluorescence spectra of tubulin with tubulin alone (black), tubulin irradiated (red), TSPP/tubulin non-irradiated (blue), TSPP/tubulin complex irradiated (teal). 


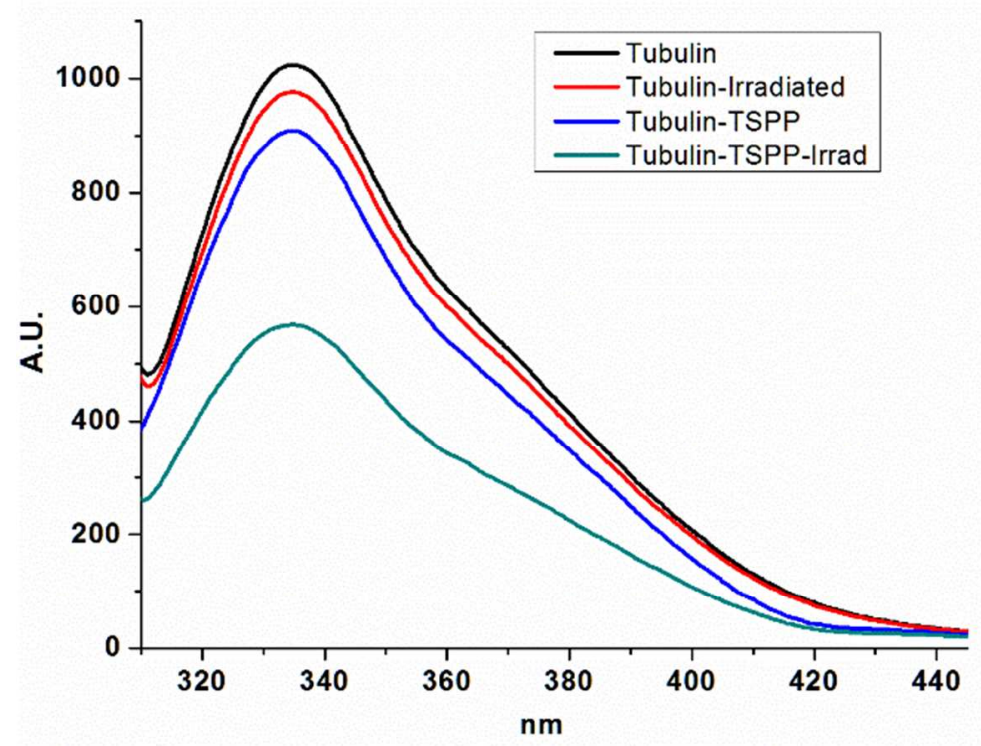

Figure S2. The effects of irradiation with the $80-\mathrm{MHz}$ source on tubulin/TSPP complex.

Fluorescence spectra of tubulin with tubulin alone (black), tubulin irradiated (red), TSPP/tubulin non-irradiated (blue), TSPP/tubulin complex irradiated (teal).

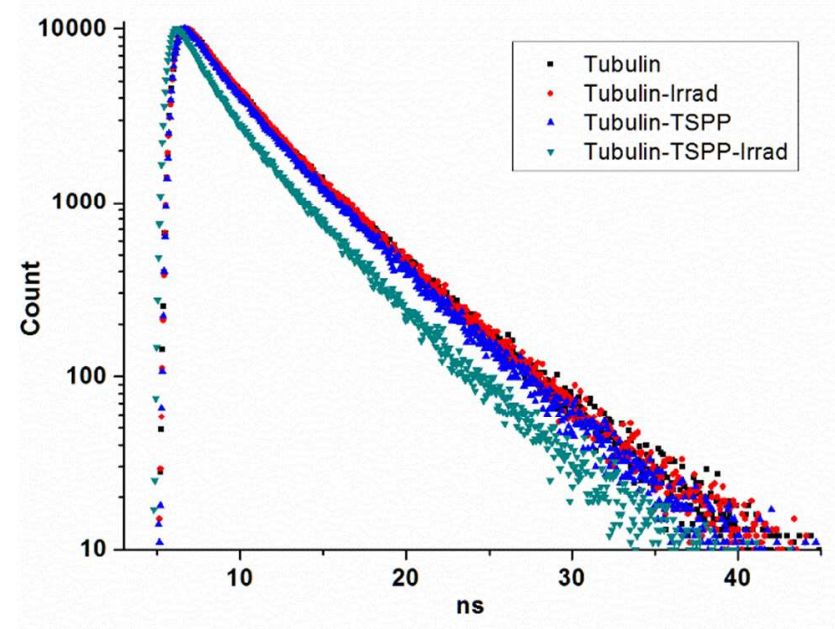

Figure S3. Fluorescence decay plots collected at $330 \mathrm{~nm}$ with an excitation source at $295 \mathrm{~nm}$. Tubulin non-irradiated (blue up triangle), tubulin irradiated (teal down triangle), Tubulin/TSPP complex (black squares), and tubulin/TSPP complex after TPA irradiation (red diamond). 